\title{
Research on the Development, Problems and Countermeasures of Humane and Sociological Science of Sports
}

\author{
Zhe Shengxiang \\ Yunnan University of Business Management, Kunming, Yunnan 650106, China
}

Keywords: China; humane and sociological science of sports (HSSS); status quo; development

\begin{abstract}
In the past ten years, humane and sociological science of sports has been attracting more and more attention in China. What is worth celebrating is that the research on humanities and sociology has also achieved satisfactory results, among which interdisciplinary and multi-disciplinary cross-disciplinary research and applied research are more obvious, making the research field and research scope gradually increase and the embryonic form of each discipline basically fixed. Despite the remarkable results, there are still some problems that need to be further improved. For examples, the basic theory of some disciplines is not profound enough; the development of sub-disciplines is not balanced; the degree of research integration is not high; and the multi-level, multi-sequence and multi-form three-dimensional net-structured discipline system has not yet been formed. In this context, this paper made an in-depth discussion and research on the development of humane and sociological science of sports in China and further strengthens the research of ontology theory, in order to create a deep atmosphere for discipline research and enhance the self-consciousness of research ontology. It is also feasible to further develop the humane and sociological science of sports with the help of research methods and achievements of foreign objects such as natural science, so as to achieve the goal of strengthening the integration of its research and sports practice.
\end{abstract}

\section{Introduction}

The time when humane and sociological science of sports (thereafter referred to as HSSS) was established in science was 1997. At that time, in addition to this discipline, three other disciplines were set up by the Academic Degrees Committee of the State Council and the State Education Commission under the first-level discipline of physical education, and they are Human Movement Science, Theory of Sports Pedagogy and Training and Science of Ethnic Traditional Sports. Since the establishment of this discipline, its development and problems has been attached great importance to and it has become an important topic to explore how to further develop and how to improve and solve the problems[1]. Therefore, on the basis of a comprehensive review of the development of HSSS in China, the author discussed and studied the above-mentioned problems to further find the direction for its development in China in the new era and to further study its sustained and rapid development.

\section{The Development of Humane and Sociological Science of Sports in China}

Between 1997 and 2007, HSSS developed at a high speed. The number of national sociological fund projects carried out or completed by Chinese scholars reached 310, and the number of social science projects planned by the State Sports General Administration reached 1073. The level and scope of various projects was unprecedented, and the intensity of state funding had never been greater. For example, since the 1980s, more than 10,000 papers related to this discipline have been published in domestic and foreign academic journals, and more than 200 academic monographs and translations have been published. Some areas have achieved rapid development and remarkable results, including sports sociology, sports economics, sports history, sports management, Olympic research and sports journalism. At present, it can be roughly known that the embryonic form of the discipline of HSSS has been formed. Generally speaking, there are several indispensable and important factors to form a new discipline: the nature of the discipline, the subject object, the scope of the discipline research, its research methods, its knowledge structure, its various functions and its main contents. First of all, there are differences in the subject nature 
of HSSS. Scholars have put forward various properties but not all have reached a consensus. At present, some scholars argue that it is a comprehensive discipline which develops mainly on the basis of two disciplines, namely sports science and humanistic sociology. Some other scholars hold that it is a cross-disciplinary combination of humanities, social marginal discipline and some other disciplines. Most researchers state that the research object of HSSS is the humanities and social phenomena in the field of sports, while a small number of researchers believe that its research object should be the sports phenomena and development rules as well as various relationships in the field.

The research methods of HSSS can be divided into three levels: general methodology, special methodology and individual methodology. The first one is a methodology system (such as philosophical thinking mode) that shows the most general laws, essence and characteristics of its research object. The special methodology is a methodology to study the special fields and methods of the research object and the special laws, essence and nature at a specific stage, such as the field of prompting sports training. The last one is a methodology system of specific research methods suitable for a specific discipline, such as the teaching method of sports technology, etc[2]. The research method of sports humanities has been much improved because of the multi-style and extensive social investigation. Researchers have clearly indicated that the knowledge structure and subject content of sports humanities are developed based on the third-level subjects. Because contents of the second-level sports disciplines are complex and extensive, some subjects relatively important are selected from them. The subject knowledge structure and subject contents which are commonly put forward by scholars in research mainly include the sports philosophy and sports history in the field of sports humanities, the sports sociology and sports economics in the field of sports sociology, etc. Scholars think that the research contents of HSSS also cover sports introduction, sports philosophy, school sports science, sports economics, comparative sports, sports aesthetics, sports psychology, operation psychology, etc. Before the 1980s, the research field of sports humanities and social science was narrow and the scope was also small in China due to the low sports level and the lack of sports knowledge. After the 1980s, with the further development of reform and opening up, sports practice has gradually expanded to political, economic, cultural, educational and other aspects, especially in the economic field. In addition, it also led to the extension of HSSS research in the fields of culture, law, communication and so on. Therefore, it is needed to promote and restrict sports in combination with the social environment and various conditions, so that the phenomena and problems in the research field can be examined and regulated under the broad social background.

It is undeniable that sports are as highly comprehensive as complex social problems or social phenomena. To be able to explore the highly comprehensive and complex problems in sports in all directions, it is necessary to conduct in-depth study and research by using the theoretical knowledge of social sciences among various disciplines. This is the scientific basis of sports science. The theoretical basis for the study of humanities and sociology is that different levels and aspects of social and cultural phenomena in sports can be introduced into many disciplines of modern humanities and social sciences. This strengthens the basic theory and cognitive ability of sports humanities research, improves the starting point and scientific research level of this discipline and expands the breadth and depth of its research. The main research aspects of HSSS include the relationship between sports and the development of economy and society, sports reform, development strategy, and so on. The scope of its research continues expanding, and it has closer ties with various disciplines such as sociology, political science and economics and their branches. The further evolution and openness of the content, form and operation mechanism of sports practice have played a key role in the expansion of HSSS research, which further makes it show increasingly distinctive characteristics of multiple subjects and multidisciplinary cross .According to the statistics, the proportion of interdisciplinary research was $37 \%$ of the total number of sports research projects, and multidisciplinary research accounted for $65 \%$ of the total number of projects. The scope of research has involved many disciplines such as philosophy, political science and economics, which makes the interdisciplinary research distinct and promotes the establishment of sub-disciplines. 
With the development of sports and the progress of science and technology, the previous inherent system structure of sports theory has become obsolete and rigid. The knowledge system of modern sports science needs to introduce new sub-disciplines into the original system, such as social sports science, sports law, sports ethics, leisure sports science, etc. Excellent research results deserve to be introduced and appreciated. Related departments and workers in China have recognized and vigorously taken action to continuously introduce foreign works in the field of HSSS. For examples, Hao Qin of Chengdu Sports Institute translated Sports News Reporting and it was published by Huaxia Publishing House in 2002; in 2003, Tsinghua Publishing House published Sports Economics, Sports Management and some other works, which are known as classic translations in sports science. The introduction of foreign excellent research results has promoted the HSSS research in China[3]. The National Social Science Foundation and the State Physical Culture Administration have shown the role of promotion in the planning and setting up of social science projects of several important areas, such as research on sports economic issues and market development, research on sports economic policy, research on sports socialization and research on sports development strategy, which makes the trend of application-oriented sports research more obvious.

In 2005, the National Guide to Sports Studies in Social Sciences showed that "in recent years, sports research has a wide field of vision, with advanced and effective research methods and clearer purpose in selecting topics, increasing the number of related topics for the economic and social development." The Social Science Program Project Guide issued by the State Physical Culture Administration also gave relevant guidance on "research content and key, difficult and hot issues in sports research".

In 2006, the thought in the National Project Guides for Sports Subject of Social Science was: "the most important subject of the year is how to grasp the difficult problems in the reform and development of sports in China and the key issues related to the healthy and coordinated development of sports, so as to put forward theoretical views and countermeasures with practical application value and operability." According to the Project Guide of the Sports Social Science Program of the State Physical Culture Administration, "The main direction of sports is to use the sports social science as the foundation to study major theoretical and practical problems in the development of sports and to launch high-level sports social science research results.”

\section{Problems in the Development of Humane and Sociological Science of Sports in China}

At present, the basic theory of HSSS discipline in China is relatively weak. Although this discipline has established its own discipline status in the sports science system, in fact it has not completely established a complete discipline system. The basic theory of HSSS has been thoroughly discussed and studied. However, in fact, the symbolic elements of the discipline HSSS (such as knowledge structure and contents of this discipline which is composed of its research scope, its research scope, its discipline nature, its discipline function and other aspects) are actually a vague concept. Moreover, researchers have not fully understood the theory contained in this discipline.

On the whole, the basic discipline theory of HSSS in China is relatively weak, and many fields rely on the introduction of relevant disciplines and learning from foreign theoretical research results, without forming their own unique theoretical system. A multi-level, multi-sequence and multi-form three-dimensional network-structured discipline system has not yet been formed. The strong development of structure and classification of modern scientific system makes the discipline system of HSSS closely follow the modern scientific system. The most fundamental thing is that it cannot be divorced from the reform and actual development of sports in China. This is a grand practical activity, with an open system and multi-level, multi-sequence and multi-form three-dimensional net-structured system, and has the potential for development.

Some scholars believe that a discipline system generally has two basic categories, namely the system academic subject and the system for teaching subject. The two systems are interrelated, complementary and mutually reinforcing with each other. Another view is that a discipline system is an organism of 
system structure and structure system. The former is the study of theoretical composition and its formation, while the latter is the study of branch composition and their relationship. The development of sub-disciplines is uneven at present. Some sub-disciplines of HSSS such as the sports law and sports ethics started late. All these disciplines need to be strengthened and improved, because the unbalanced development will limit the further development of sports humanities and social sciences in China. In addition, the degree of research integration is not high enough, which may result in limitations for the individual research and research on a single discipline. To form a complete theory, research should be carried out in an interdisciplinary and joint way, rather than a single, independent and uncoordinated way.

\section{Strategies for the Development of Humane and Sociological Science of Sports in China}

First of all, it is necessary to strengthen the ontological study of HSSS. The study of the own theory of a discipline is very important. In addition to grasping contents such as subject foundation and values, it is also needed to actively explore and develop some new subjects. Further practical analysis and application is an important step to strengthen the research on ontology theory of HSSS and promote its modern development. It can be started from two aspects: its theoretical system and its disciplinary system. The second is to create a strong atmosphere for the study of HSSS. An excellent discipline research team is the key to forming a good environment[4]. In China, it is significant to form a reasonable academic and innovation team, and all departments should carry out academic activities of discipline research in an all-round way, so that everyone can learn and progress together and jointly study. The state should vigorously support and reward individuals and teams obtaining outstanding innovative achievements in research. There is also a need to improve the self-awareness of HSSS research. This means that the researchers must have research spirit, which requires them to carry out research actively with the belief of continuous transcendence. It plays an important role in the development of HSSS. Then, researchers are expected to be good at developing HSSS with the help of research methods and achievements of natural science, and make use of natural phenomena and human phenomena to conduct in-depth research. This combination of qualitative and quantitative research is helpful to complete people's understanding of the phenomenon of sports humanities. Finally, it is significant to realize the integration of HSSS research and sports practice. All the contents reflected in the HSSS theory can only be proved and fed back by sports practice. Therefore, perfecting the development form of HSSS should be carried out in all aspects and with sports practice.

\section{Conclusion}

This paper described the development of humane and sociological science of sports in China and carried out in-depth discussion and study, which will help to strengthen the research of the ontology theory, create a deep atmosphere for discipline research, improve the self-consciousness of research ontology, and further strengthen the development of this discipline with the help of research methods and achievements of natural science.

\section{Reference}

[1] Yang Qianxia. A Research on Current Situation, Problems and Countermeasures of Sport Humanistic Sociology in China [J]. Reading and Writing <DuYuXie>, 2017, 14(15).

[2] Pang Zhibin. Research Status and Development Direction of Humane and Sociological Science of Sports in China [J]. Contemporary Sports Technology, 2017, 7(3).

[3] Zhou Hongsong. Research Status and Development Direction of Humane and Sociological Science of Sports in China [J]. New Education Era: Teacher's Edition, 2017(26). 